\title{
Work in Progress: A Multi-Faceted Laboratory Module in Cardiovascular Fluid Mechanics to Develop Analysis and Evaluation Skills in Biomedical En- gineering Undergraduates
}

\section{Dr. Jeffrey A. LaMack, Milwaukee School of Engineering}

Dr. LaMack teaches full-time in the Biomedical Engineering program in the Electrical Engineering and Computer Science Department at the MIlwaukee School of Engineering (MSOE). His areas of specialty include biophysical transport phenomena, biocomputing, physiology, and engineering design. Dr. LaMack holds a Ph.D. in Biomedical Engineering from Duke University, and he is an alumnus of the Biology Scholars Program of the American Society of Microbiology. Prior to becoming focused on engineering education, his research interests included hemodynamics and the study of how vascular cells respond to fluid forces and its implications in vascular pathologies.

\section{Dr. Charles S. Tritt, Milwaukee School of Engineering}

Dr. Tritt has been the director of the Biomedical Engineering program at the Milwaukee School of Engineering (MSOE) since 2009. He has been teaching at MSOE since 1990. His Ph.D. is in Chemical Engineering from the Ohio State University as is his B.S. degree. He holds an M.S. in Biomedical Engineering, also from Ohio State. His research interests include biomedical applications of mass, heat and momentum transfer; medical product and process modeling; biomaterials; and entrepreneurship, innovation and commercialization in engineering education. 


\section{Work in Progress: A Multi-Faceted Laboratory Module in Cardiovascular Fluid Mechanics to Develop Analysis and Evaluation Skills in Biomedical Engineering Undergraduates}

Today's workforce demands engineers with skills that far exceed theoretical knowledge in discrete engineering subjects. Among the desirable traits for engineers are a thorough understanding of engineering tools and the ability to integrate knowledge from different disciplines. ${ }^{1,2}$ Undergraduate capstone design experiences are largely developed to provide a setting for the attainment of these skills, and many have demonstrated positive outcomes in these areas. ${ }^{3,4}$ However, it remains a challenge to provide students with opportunities to hone these skills, particularly those based in the highest levels of Bloom's Taxonomy of synthesis and evaluation, elsewhere in the curriculum. The junior-level undergraduate biomedical engineering laboratory module described here attempts to do this by asking students to use three distinct engineering approaches toward a common problem in a multidisciplinary laboratory course.

\section{Multidisciplinary Laboratory Course Description}

A set of three one-trimester multi-disciplinary laboratory courses have recently been developed and are being implemented in the biomedical engineering curriculum at the Milwaukee School of Engineering. ${ }^{5}$ Each of these laboratories is meant to connect concepts from two concurrent didactic courses, as well as to give students opportunities to use various engineering tools to solve problems based on the theoretical concepts derived in the lecture courses. One of these laboratory courses serves as the bridge for concurrent courses in physiology and biotransport. The laboratory module described here, which is part of that particular course, specifically addresses concepts of cardiovascular fluid mechanics, as it directs students to explore the impact of the presence of a catheter in a typical large artery.

Overview of the Laboratory Module

The primary education objectives of the laboratory module are for students to:

1. Gain hands-on experience using the tools of fluid system analysis, including pumps, flow and pressure measurement devices, and computational fluid dynamics (CFD) software.

2. Evaluate various engineering approaches toward analyzing the fluid mechanical and physiological effects of a catheter on arterial flow.

Specific topics in the concurrent lecture courses to be reinforced with this laboratory module include: for the physiology course, basic pressure-flow relationships in the vascular tree; and in the biotransport course, principles of flow field analysis.

To address the objectives, a three-part laboratory module was developed. Prior to advancing to this particular module, additional laboratories in the course require that students create and characterize a fluid with the rheological properties of blood and become acquainted with the use of a pump and flow sensor by calibrating the flow sensor for a range of flow rates. In the physiology course, students will have completed the unit on cardiovascular physiology, including application of Poiseuille's Law in the vasculature and control of systemic circulation. In the biotransport courses, students will have completed units on the phenomena of fluid flow 
and internal flow, and they will be in the process of studying the use of the Navier-Stokes equations for analysis of flow fields.

The laboratory is broken down into analytical, experimental, and computational approach components; each allocated to one weekly three-hour laboratory period. An introduction is provided to students on the clinical relevance of the situation involving the placement of a catheter in a large artery. Reasons for the need to know the pressure-flow relationship for a catheter-containing artery, as well as the force imparted on the catheter by the flowing blood, are discussed. At the very beginning, students are instructed to make observations regarding advantages, disadvantages, and assumptions required for each mode of analysis.

Students work in teams (ideally of three) throughout the three-week lab module. Each student is instructed to keep record of his or her work using an engineering log book. Deliverables include a team report and submission of individual log books upon completion of the module.

Description of Individual Approaches

The initial approach students are to take is an analytical approach. While students will have seen the derivation of Poiseuille flow (i.e., pipe flow) from first principles in class, here they are asked to derive the velocity profile for a tube containing a centered catheter by applying a series of assumptions to the continuity and Navier-Stokes equations for cylindrical flow. These assumptions include steady, incompressible, axisymmetric, fully-developed flow of a Newtonian fluid with no circumferential velocity component. They are asked to solve the equations for the axial velocity, $\mathrm{v}_{\mathrm{z}}$, and given the following result to pursue:

$$
v_{z}=\frac{1}{4 \mu} \frac{\Delta P}{L}\left[r^{2}-R_{1}^{2}+\frac{\left(R_{1}^{2}-R_{2}^{2}\right) \ln \left(r / R_{1}\right)}{\ln \left(R_{2} / R_{1}\right)}\right],
$$

where $\Delta \mathrm{P} / \mathrm{L}$ is the pressure drop per length, $\mu$ is the Newtonian viscosity, $r$ is the radial coordinate, and $\mathrm{R}_{1}$ and $\mathrm{R}_{2}$ are the fixed radii of the catheter and inner wall of the blood vessel, respectively. Based on the theoretical velocity profile, students are then asked to perform the following:

- Use Excel to plot the velocity profile versus radius for two different catheter radii, as well as in the absence of a catheter.

- Use numerical integration and Excel's equation solver to compute the pressure drop per length for a prescribed flow rate, $\mathrm{R}_{1}$ and $\mathrm{R}_{2}$, and using the viscosity that the students measured for fluid they created to mimic blood.

- Calculate shear stress and drag force acting on the catheter based on the velocity profile (eq. 1), as well as using a near-wall velocity gradient approximation.

A sample velocity profile is given in Figure 1a.

The second approach employed by students is an experimental one. Here, students are supplied with a variety of equipment, most of which they had used in a prior lab module, including a roller pump and pulse dampener (Cole-Parmer, Vernon Hills, IL), a rotary liquid flow sensor (Cole-Parmer) and digital multimeter, a differential pressure transducer and strain gage meter (Omega Engineering, Inc., Stamford, CT), a catheter introducer sheath set (B. Braun Medical 
Inc., Bethlehem, PA), and a variety of tubing supply, fittings, and stopcocks. Given a basic schematic for a flow loop that incorporates the flow sensor and pressure taps leading to the transducer, students are instructed to devise an experimental plan to measure the pressure drop per length for a segment of tubing containing a wire (inserted via the catheter introducer set), matching the dimensions and other parameters used in the analytical approach. In particular, students are cautioned that they must account for minor losses arising from the pressure taps at either end of the arterial model. The goal is for students to discover that by repeating the pressure drop measurement for two different tubing lengths, they can determine the minor loss and subtract it off. Students use their blood model as the working fluid in this experiment.

The final approach taken for this problem is the use of CFD. The students, who have no previous experience with computational methods, are provided with a brief description of the approach and a tutorial that walks through the solution of simple pipe flow using COMSOL Multiphysics (COMSOL, Inc., Burlington, MA). The tutorial includes exploration of mesh scheme and density sensitivity, as students implement different meshes that produce increasingly accurate solutions. Using identical dimensions and parameters used in the first two approaches, students are then asked to create a geometry for a blood vessel containing a catheter, mesh it, solve it, and determine the pressure drop per length and drag force acting on the catheter. A sample solution depicting the velocity distribution at the tube outlet is shown in Figure $1 \mathrm{~b}$.

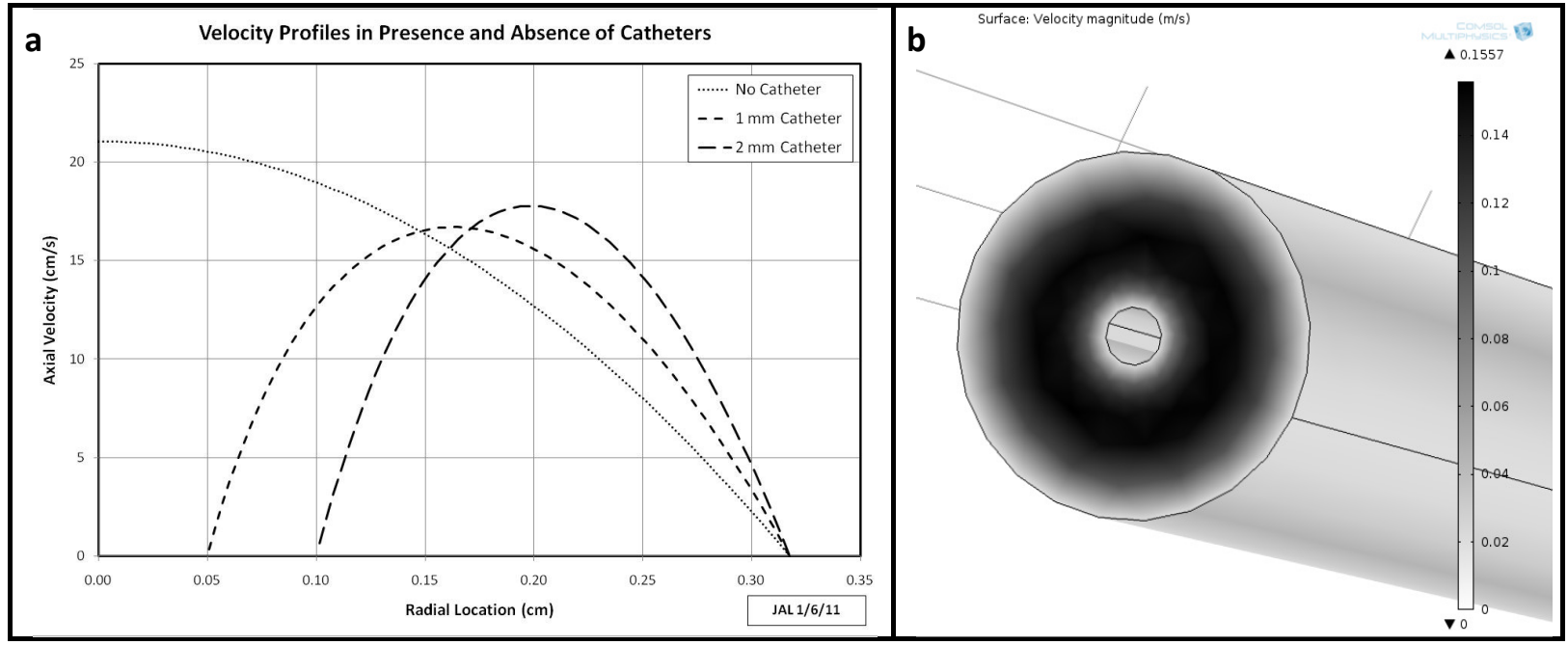

Figure 1: Sample solutions of radial velocity profiles for arterial blood flow, (a) in the presence and absence of a catheter (two sizes) based on the analytical solution for this flow field and (b) in the presence of a catheter based on the computational fluid dynamics approach.

\section{Assessment of Student Work}

As described above, two separate deliverables are associated with this project. The first, which is weighted more heavily, is a team report. The report has specific requirements for each aspect of the project, as well as the following requirements to evaluate the various approaches:

Report Requirement: An evaluation of the applicability of each of the three models you used in this lab. Be sure to address the following:

- What assumptions were common to all three models? 
- What assumptions were unique to each of the three models? How much error would you expect each assumptions to introduce (you can be subjective here--small or large amounts of error, etc.)?

- Apart from errors arising from the various assumptions, what other sources of error attributed to calculations and/or measurements for each model? How much relative error do you thing each might introduce?

- What, specifically, do you think accounted for discrepancies between the analytical and CFD results?

- Taking everything into account, for which of the three models would you expect the calculations and/or measurements to best represent its corresponding physiological situation and why?

A grading rubric is used to grade the report that includes individual assessment of the requirements above, as well as the various technical requirements for each approach. In addition, each student's log book is collected and graded. A separate rubric assesses each student's record-keeping ability, logic of analysis, and interpretation of results.

Results of the Laboratory Module and Assessment of Its Impact

Thus far, the laboratory has run three years. The first two years, the lab ran smoothly, while in the third, a number of equipment issues arose with the experimental approach that impacted the ability of the students to evaluate it against the other two approaches. Plans are in place to implement a formal, IRB-approved assessment of the impact of the module during the next academic year. The assessment will involve, in addition to analysis of performance based on the grading rubrics, a pre- and post-test designed to measure: (1) students' understanding of basic concepts in fluid mechanics; (2) students' ability to apply fluid mechanics concepts to pathophysiology involving alteration of flow; and (3) whether students are able to propose valid approaches to measuring or estimating fluid mechanical behavior in physiological systems. Students will also be surveyed on the extent to which they feel they are able to evaluate different engineering approaches before and after the course, and senior design instructors will be asked to assess students' abilities to conduct proper analysis to make decisions based on thorough evaluation, rather than trial and error, in their respective design projects. The developers of the laboratory expect to see increased evaluation and synthesis skills of students following the course and hope this is reflected in their ability to make informed design decisions in their capstone design course.

[1] Splitt, F. G. "The Challenge to Change: On Realizing the New Paradigm for Engineering Education". Journal of Engineering Education, 2003, vol. 92, no. 2, pp. 181-187.

[2] Fromm, E. "The Changing Engineering Educational Paradigm". Journal of Engineering Education, 2003 , vol. 92, no. 2, pp. 113-121.

[3] Phillips, J.R. and Gilkeson, M.M. "Twenty-Five Years of Experience in a Clinical Approach to Engineering Design”. Proceedings of the 1990 Frontiers in Engineering Conference, Vienna, Austria, July 1990, pp. $343-345$.

[4] Miller, R., Olds, B. “A Model Curriculum for a Capstone Course in Multi-Disciplinary Engineering Design”. Journal of Engineering Education, 1994, vol. 83, no. 4, pp. 311-316.

[5] Gassert, J.D., Imas, O., LaMack, J., Fennighoh, L., Gerrits, R., Schlick, N., Tritt, C. "Cross-Disciplinary Biomedical Engineering Laboratories and Assessment of their Impact on Student Learning". Proceedings of the 2011 ASEE Annual Conference, Vancouver, BC, Canada, AC 2011-2848. 